\title{
Infectious pneumonia in immunocompetent patients: updates in clinical and imaging features
}

\author{
Kyung Soo Lee ${ }^{1}$, Tae Jung Kim ${ }^{1}$, Kyong Ran Peck ${ }^{2}$, Joungho Han ${ }^{3}$ \\ ${ }^{1}$ Department of Radiology, Samsung Medical Center, Sungkyunkwan University School of Medicine, Seoul, Korea \\ ${ }^{2}$ Division of Infection, Department of Internal Medicine, Samsung Medical Center, Sungkyunkwan University School of Medicine, \\ Seoul, Korea \\ ${ }^{3}$ Department of Pathology, Samsung Medical Center, Sungkyunkwan University School of Medicine, Seoul, Korea
}

Received: July 13, 2018

Revised: August 18, 2018

Accepted: August 20, 2018

Corresponding author:

Kyung Soo Lee

Department of Radiology,

Samsung Medical Center,

Sungkyunkwan University

School of Medicine, 81 Irwon-ro,

Gangnam-gu, Seoul 06351, Korea

Tel: +82-2-3410-2511

E-mail:

kyungs.lee@samsung.com

\begin{abstract}
Morphologically pneumonia is usually classified into lobar pneumonia, bronchopneumonia, and interstitial pneumonia. Chronic pneumonia is also a kind of pneumonia in immunocompetent and mildly immunocompromised patients. Specific organisms may be involved in community-acquired pneumonia (CAP) according to patients' age or underlying conditions. The organisms involved in CAP are different from those in hospital-acquired pneumonia. Mixed pneumonia includes septic pneumonia, lung abscess, and focal organizing pneumonia. The role of imaging in pneumonia includes the detection or exclusion of the presence of pneumonia, narrowing down of differential diagnosis of the pneumonia from other lung conditions, planning of further diagnostic procedure, and the assessment of treatment response with follow-up studies. New drugs for pneumonia are expected to open a door widely for antibiotic treatment for various pneumonias.
\end{abstract}

Keywords: Anti-bacterial agents; Computed tomography; Immunocompetent; Management; Pneumonia
This is an Open Access article distributed under the terms of the Creative Commons Attribution Non-Commercial License (http:// creativecommons.org/licenses/ by-nc/4.0/).

\section{INTRODUCTION}

Pneumonia refers to lung inflammation caused by bacterial or viral infection and pathologically to the condition where the alveolar spaces are filled with pus and the lungs become solid. The inflammation may affect the both lungs, one lung or only the certain lobe(s).

The pneumonia can occur in young and healthy people, but the condition is most dangerous for elderly people, infants, and patients with other disease(s), and those (immunocompromised patients) with impaired immune systems. Pneumonia affects approximately 450 million people globally ( $7 \%$ of the population) and results in about 4 million deaths per year [1]. It occurs about five times more frequently in the developing countries than in the developed countries. Viral pneumonia accounts for about 200 million cases. In the United States, approximately one million people are treated in the hospital for pneumonia in each year, and about 50 thousand peo- 
ple die of the disease. The disease is the leading cause of death owing to infection in children $<5$ years of age worldwide, and pneumonia and influenza together are ranked as the eighth leading cause of death in the United States [2].

The pneumonia start with symptoms similar to those of cold and flu, then a high fever, chills and cough, and sputum follow. Other symptoms include chest pain, tachycardia, nausea and vomiting, headache, muscle pain, confusion or delirium, or skin discoloration and cyanosis. These symptoms may vary depending on the type of pneumonia and other underlying conditions.

The clinical, morphologic, and pathologic findings have been well known. However, still it is difficult to make an accurate diagnosis of pneumonia particularly regarding causative organisms related to the morphologic pneumonia. Meanwhile, the incidence of chronic pneumonia is increasing as a manifestation of pneumonia in mild immunocompromised patients. The aim of this review was to update the information on pneumonia focused on imaging features and new antibiotic agent in the diagnosis and treatment of pneumonia in the era of personalized medicine.

\section{Settings of pneumonia development Community-acquired pneumonia}

Community-acquired pneumonia (CAP) is acquired in the community, outside of health care facilities. Compared with healthcare-associated pneumonia (HCAP), it is less likely to involve multidrug-resistant bacteria. Although the latter are no longer rare in CAP, they are still less likely. The mortality of CAP in cases of outpatients is reported to be $1 \%$ to $5 \%$, whereas that in CAP necessitating hospitalization is reported to be as high as $25 \%[3,4]$.

\section{Healthcare-associated pneumonia}

HCAP is an infection associated with recent exposure to the health care system, including hospital, outpatient clinic, nursing home, dialysis center, chemotherapy treatment, or home care $[3,4]$.

\section{Hospital-acquired pneumonia (also called nosocomial pneumonia)}

Hospital-acquired pneumonia (HAP) is acquired in a hospital, specifically, pneumonia that occurs 48 hours or more after admission, which was not incubating at the time of admission. It is likely to involve hospital-acquired infections, with higher risk of multidrug- or extensively drug-resistant pathogens. The HAP may contain HCAP and ventilator-associated pneumonia [5].

\section{Ventilator-associated pneumonia}

Ventilator-associated pneumonia occurs in people breathing with the help of mechanical ventilation. Ventilator-associated pneumonia is specifically defined as pneumonia that arises more than 48 to 72 hours after endotracheal intubation.

\section{Incidence of pathogens}

CAP in patients of $<60$ years of age and without comorbidity Streptococcus pneumoniae, Mycoplasma pneumoniae, respiratory viruses, Hemophilus influenzae, Chlamydia pneumoniae, and Legionella species are the most frequent organisms involved [6].

\section{CAP in patients of $>60$ years of age or with comorbidity}

$S$. pneumoniae, respiratory viruses, $H$. influenzae, aerobic gram-negative bacilli, and Staphylococcus aureus are the most frequently involved organisms [7].

Incidence of pathogens in pneumonia requiring hospitalization

They are S. pneumoniae, H. influenzae, polymicrobial organisms, anerobic gram-negative bacilli, Leginella species, S. aureus, $C$. pneumoniae, and respiratory viruses.

\section{Hospital-acquired pneumonia}

Gram-negative organisms including Pseudomonas aeruginosa, enterobactor species, Klebsiella pneumoniae, Escherichia coli, and $\mathrm{H}$. influenzae account for approximately $60 \%$ of involved organisms; gram-positive bacilli including S. aureus and S. pneumoniae are involved in about $20 \%$ of infection; and fungi are involved approximately in $5 \%$ of HAP.

\section{CLASSIFICATION}

\section{Morphologic classification}

Pulmonary infection can also be classified into several radiologic and pathologic patterns according to its morphologic features. The three most common patterns are lobar pneumonia, bronchopneumonia, and interstitial pneumonia. Less common forms of infection include cellular bronchiolitis, septic embolism, miliary infection, and lung abscess. Because less common forms of infectious condition shall be dealt with the subheading of mixed infection, the three most common patterns are described here.

\section{Lobar pneumonia}

Lobar pneumonia is characterized histopathologically by fill- 
ing of alveolar airspaces by an exudate of edema fluid and neutrophils. This filling is usually uniform within the affected lung and typically extends across pulmonary segments. The consolidation usually begins in the periphery of the lung adjacent to the visceral pleura, and spreads via interalveolar pores and small airways centripetally, sometimes to involve the entire lobe. Bronchi that remain filled with gas and become surrounded by the expanding inflammatory exudate are often seen as air bronchograms on computed tomography (CT) scans. The most common causative organisms are $S$. pneumoniae, K. pneumoniae (Fig. 1), and Legionella pneumophila.

On CT scans, homogeneous airspace consolidation involving adjacent segments of a lobe is the predominant finding. On high-resolution computed tomography (HRCT), areas of ground-glass opacities (GGOs) denoting incomplete filling of alveoli can be seen adjacent to the airspace consolidation
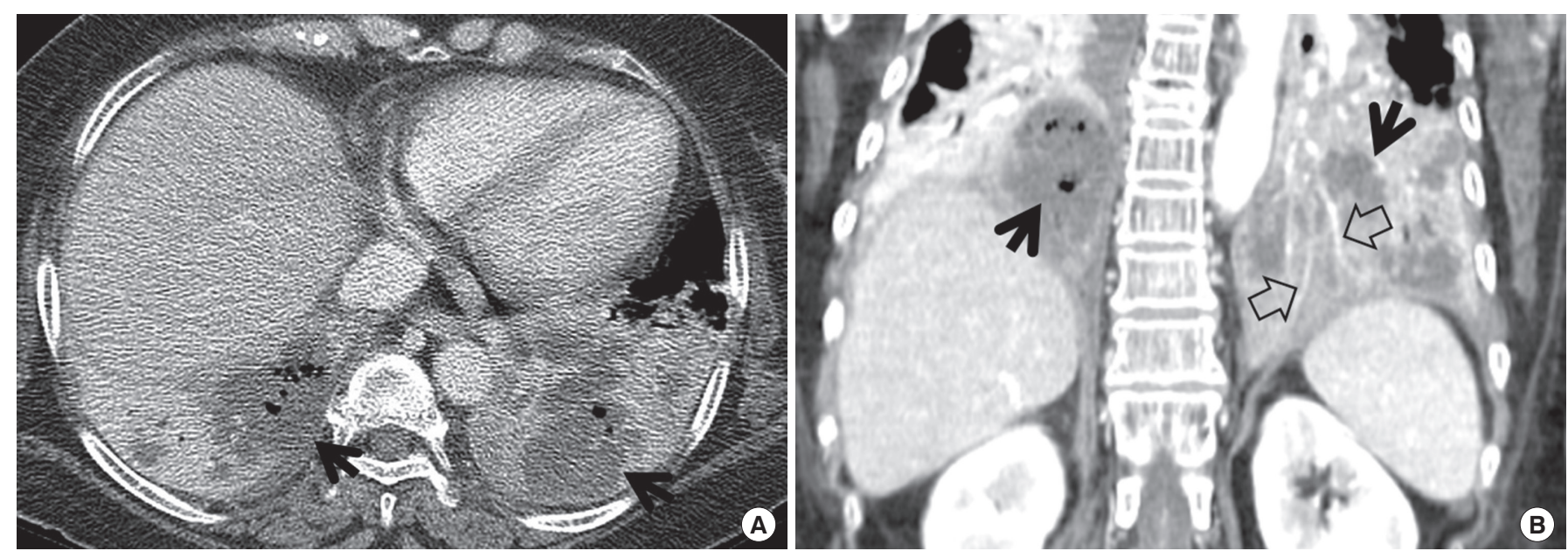

Fig. 1. Klebsiella pneumonia in a 62-year-old woman. (A) Mediastinal window image of enhanced computed tomography (CT) scan (2.5-mmsection thickness) obtained at level of liver dome shows dense lobar consolidation in both lower lobes containing multi-focal areas of necrotic low-attenuation areas (arrows). (B) Coronal-reformatted (2.0-mm-section thickness) image demonstrates necrotic (arrows) lobar consolidation involving both lower lobes. Also note CT-angiogram signs (open arrows).

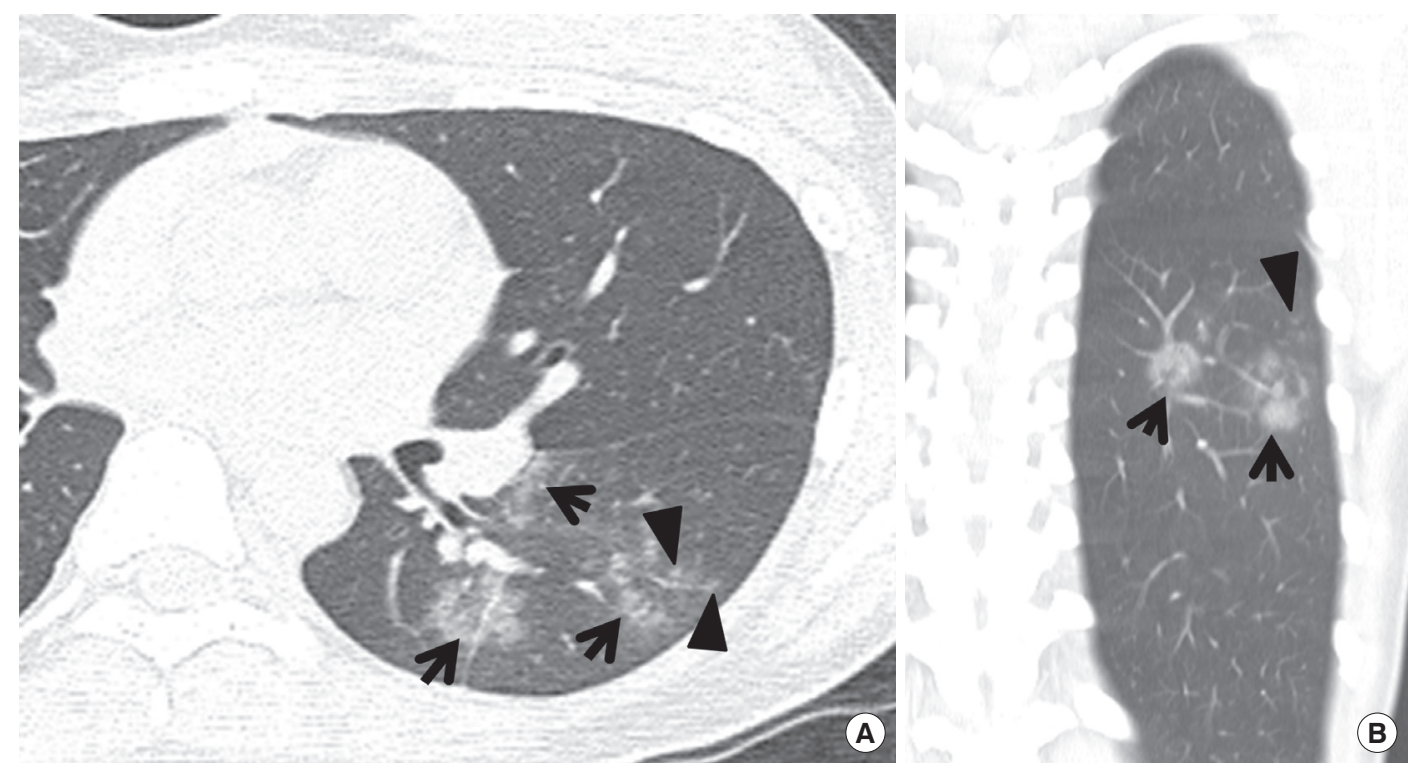

Fig. 2. Computed tomography (CT) morphology of bronchopneumonia in community-acquired pneumonia in a 22-year-old woman. (A) Lung window image of CT scans (2.5-mm-section thickness) obtained at level of left basal trunk shows bronchopneumonia comprised of variable-sized ground-glass opacity lesions (arrows) and small ground-glass opacity nodules (arrowheads) along bronchovascular bundles. Please note poorly-defined nature of lesions. (B) Coronal-reformatted (2.0-mm-section thickness) image demonstrates same-natured ground-glass opacity lesions (arrows) and nodules (arrowhead). 


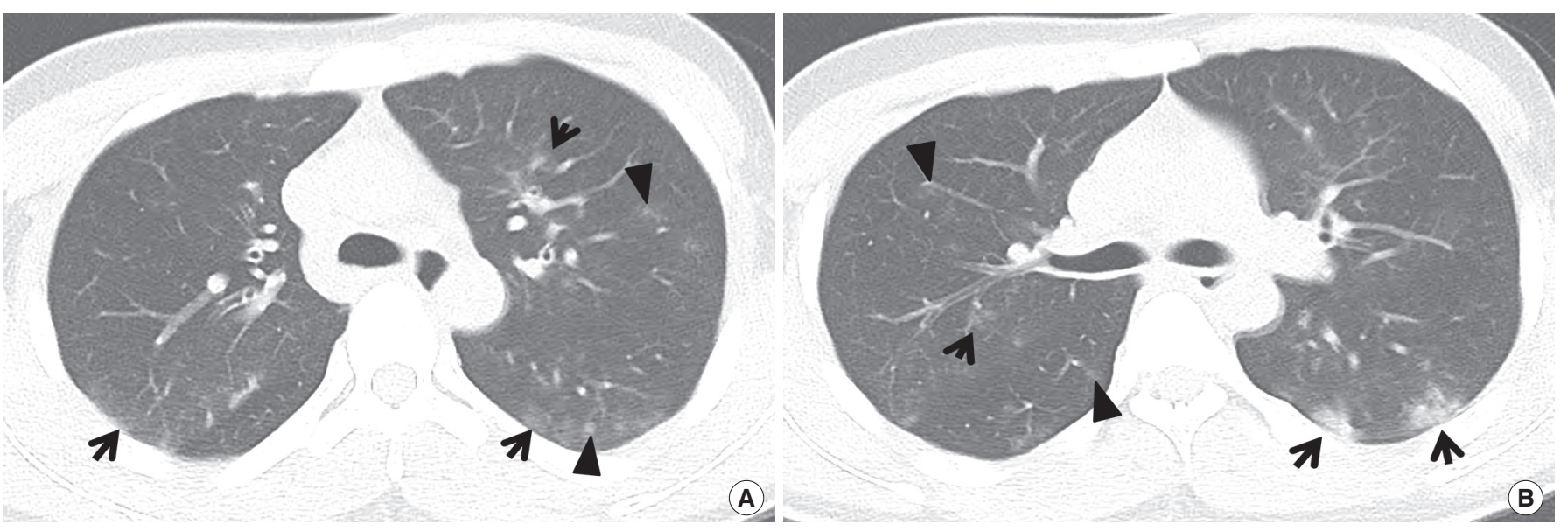

Fig. 3. Computed tomography (CT) morphology of bilateral bronchopneumonia in community-acquired pneumonia in a 20-year-old man. (A, B) Lung window images of CT scans (2.5-mm-section thickness) obtained at levels of azygos arch (A) and right upper lobar bronchus (B), respectively, depict ground-glass opacity lesions (arrows) in subpleural or subfissural location or along bronchovascular bundles and ground-glass opacity nodules (arrowheads) along bronchovascular bundles in both lungs. Also note poorly-defined nature of abnormalities.

(Fig. 1) [8]. The consolidation typically extends across lobular and segmental boundaries.

Segmental or lobar form of tuberculous pneumonia may occur in adults; in acquired immune deficiency syndrome (AIDS) or non-AIDS immunocompromised patients including pregnant women, elderly, diabetes, alcoholics, and transplanted patients. Multiple small cavities may be shown within the consolidative lesion $[9,10]$.

\section{Bronchopneumonia}

Bronchopneumonia is characterized pathologically by patchy, predominantly peribronchiolar inflammation. The reason why this localization is different from lobar pneumonia is unclear, but may be related to relatively less abundant edema formation (associated with more difficult spread of infection within the lung) and more virulent organisms (resulting in greater tissue destruction) in bronchopneumonia. Although initially patchy, progression of disease may result in lobular and segmental consolidation. The main causative organisms are $S$. aureus, $H$. influenzae, $P$. aeruginosa, and anaerobic bacteria.

Characteristic findings on HRCT include centrilobular small nodules and branching nodular structures, airspace nodules, and multi-focal lobular consolidation or GGO (Figs. $2,3)[8]$. The small nodules and branching linear opacities result in an appearance resembling a tree-in-bud and are related to the presence of the inflammatory exudate in the lumen and walls of membranous and respiratory bronchioles and the lung parenchyma immediately adjacent to them. This appearance is similar to cellular bronchiolitis caused by viruses, $M$. pneumoniae and Chlamydia [8,11]. Unlike the latter (atypical pneumonias), however, the tree-in-bud pattern in bacterial bronchopneumonia usually comprises only a small proportion of the HRCT abnormalities. In addition, thickening of bronchovascular bundles is more frequently observed in atypical pneumonia, in which individual opacity tend to be distributed at the inner zone in addition to the middle and outer zones and be recognized smaller than those of bacterial pneumonias [8].

Pulmonary tuberculosis (TB) also presents with prototypical morphologic dense (much denser [Fig. 4] than ordinary bacterial pneumonia) bronchopneumonia. On CT, the lesion consists of tree-in-bud pattern, acinar nodule, lobular consolidation, and cavitating or noncavitating nodules or subsegmental or segmental consolidation. Bronchial wall thickening may be associated [9]. Clustering of tree-in-bud sign may result in CT-galaxy sign (Fig. 4).

\section{Interstitial pneumonia}

Interstitial pneumonia is characterized histologically by a mononuclear inflammatory cell infiltrate in the alveolar septa and interstitial tissue surrounding small parenchymal vessels (Fig. 5). The most common causes are M. pneumoniae, viruses, and Pneumocystis jirovecii.

The followings are common pathologic findings in viral pneumonia: viruses can result in several pathologic forms of low respiratory tract infection including tracheobronchitis, cellular bronchiolitis, and pneumonia. Because viral organ- 

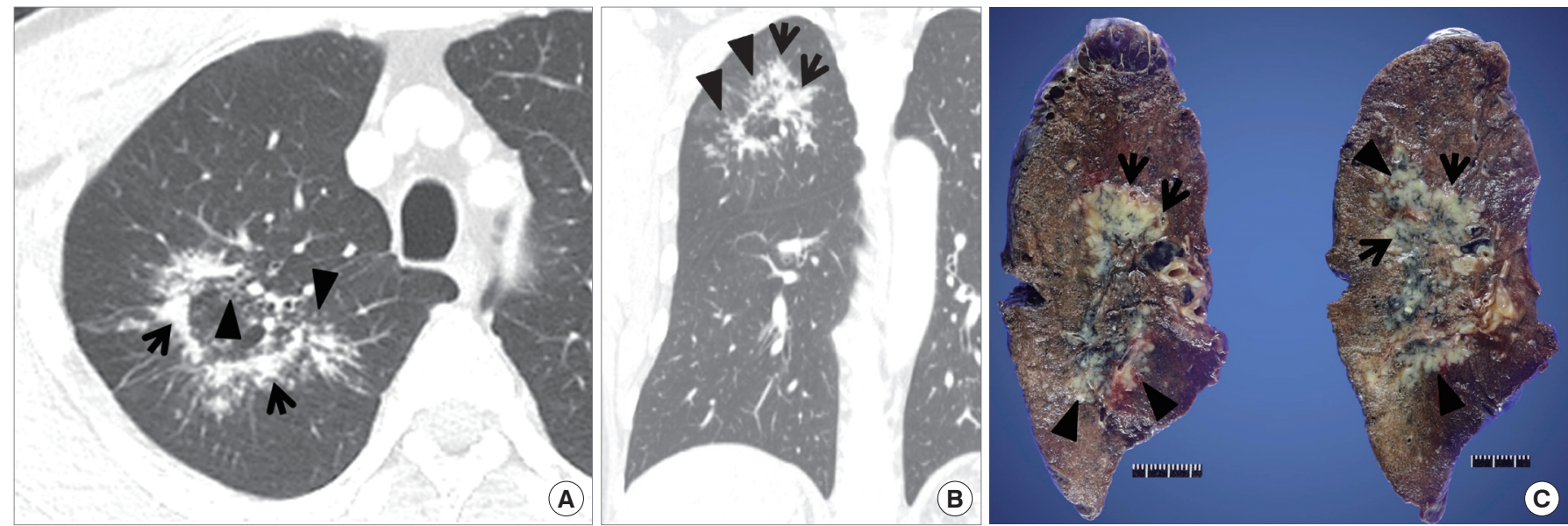

Fig. 4. Computed tomography (CT) morphology of dense bronchopneumonia in active pulmonary tuberculosis in a 56-year-old man. (A) Lung window image of CT scan (2.5-mm-section thickness) obtained at level of great vessels arising from aortic arch demonstrates combined lesions of dense opacity (arrows) and branching small nodular lesions (arrowheads) in right upper lobe. Please note dense nature of lesions differently from lesions of community-acquired pneumonia (Figs. 2, 3). (B) Coronal reformatted (2.0-mm-section thickness) image depicts same-natured dense opacity lesions (arrows) and branching small nodules (arrowheads). (C) Gross pathologic specimen discloses tuberculosis lesions comprised of dense consolidation (arrows) and small branching nodules (arrowheads).

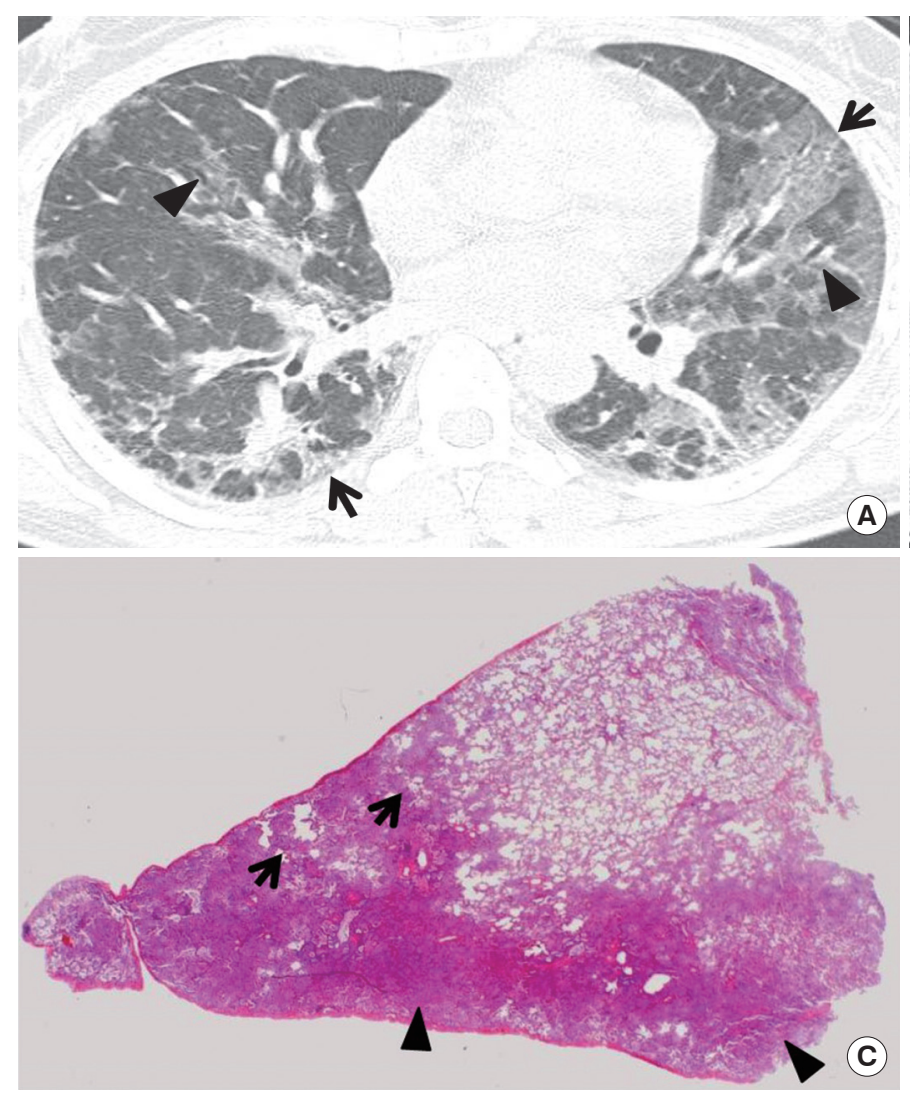

isms replicate within tissue cells, the most prominent histologic changes are observed in the epithelium and adjacent interstitium. In tracheobronchitis, airway walls are congested and lumen contains mononuclear cell infiltrates. Degeneration and desquamation of the epithelial cells are seen. Cellular

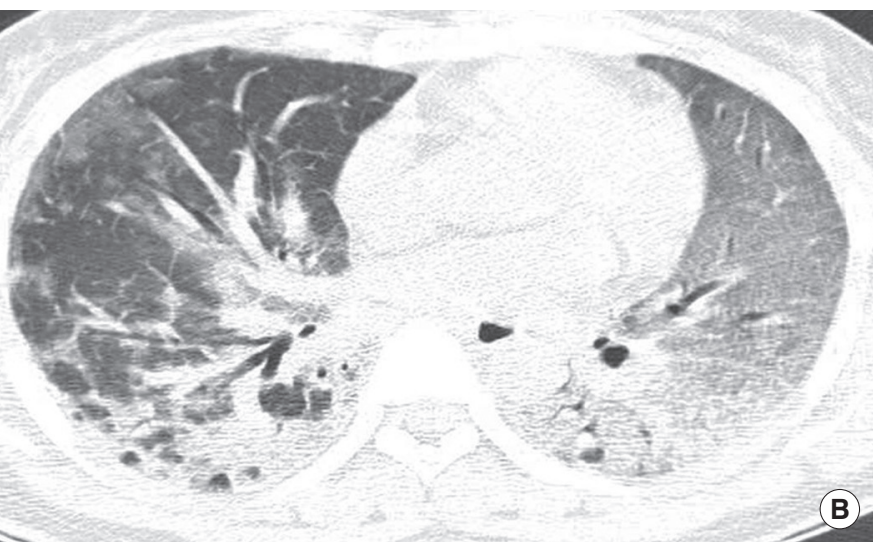

Fig. 5. Interstitial-pneumonia pattern in adenoviral pneumonia in a 44-year-old immunocompetent woman. (A) Lung window image of computed tomography (CT) scan (2.5-mm-section thickness) obtained at level of right inferior pulmonary vein shows patchy areas of parenchymal opacity distributed along bronchovascular bundles (arrowheads) or subpleural lungs (arrows). (B) CT scan obtained at level of basal trunk demonstrates more extensive parenchymal opacity in both lungs. (C) Low-magnification photomicrograph (H\&E stain) of surgical lung biopsy specimen obtained in superior segment of right lower lobe discloses multifocal consolidation (arrows) with intraalveolar fibrin exudate and interstitial widening (arrowheads) owing to fibroblastic proliferation and lymphocyte infiltration.

(inflammatory) bronchiolitis, particularly important in children, appears with epithelial necrosis, neutrophilic exudate in the airway lumen, and predominantly mononuclear infiltrates in its wall. Parenchymal involvement, the pneumonia, starts with the involvement of the lung adjacent to the terminal and 
respiratory bronchioles; however, extension throughout the lobule may occur (Fig. 5). Rapidly progressive pneumonia may occur particularly in the elderly and in immunocompromised patients where the lungs histologically show diffuse alveolar damage (Fig. 5) comprising interstitial lymphocyte infiltration, airspace hemorrhage, edema and fibrin, type 2 cell hyperplasia, and hyaline membrane formation [12].

Viral pneumonia manifest radiologically as poorly-defined nodules (airspace nodules of 4 to $10 \mathrm{~mm}$ in diameter) and patchy areas of peribronchial GGO and airspace consolidation (Fig. 5). Because of the associated cellular bronchiolitis, hyperinflation is commonly present [12-14]. Progressive pneumonia shows the rapid confluence of consolidation leading to diffuse alveolar damage, consisting of homogeneous or patchy unilateral or bilateral airspace consolidation and GGO or poorly-defined small centrilobular nodules [12].

\section{Pattern approach in pneumonia}

The con of pattern approach lies in the fact that such approach helps to design targeted antibiotic treatment or experimental and presumed antibiotic treatment. However, the pros of such approach may include (1) overlap of causative organisms causing a certain pattern of pneumonia, (2) changes in presenting pattern of pneumonia according to patients' immunity, and (3) changes in the manifesting pneumonia pattern related to pre- or co-existing lung disease.

\section{Pattern approach in diffuse pneumonia}

CT parenchymal lung abnormalities in diffuse pneumonia may be classified into three main patterns according to the distribution and patterns of the lung abnormalities; cryptogenic organizing pneumonia (OP) (Fig. 6), acute interstitial pneumonia (AIP) (Fig. 5), and bilateral extensive bronchopneumonia. This classification may help stratify patients with viral pneumonia in terms of their prognoses. In a particular viral pneumonia (influenza A [H1N1] pneumonia), patients with bronchopneumonia pattern have a tendency to belong to the mild illness group, whereas patients with AIP pattern show more severe clinical course in their outcome $[8,15,16]$. According to one study, CT findings in patients having a mild pneumonia course consist mainly of inflammatory lesions involving large or small airways with focal centrilobular small nodules with tree-in-bud sign and bronchiolar wall thickening [17]. Fatal cases of the same pneumonia present as areas of consolidation with or without GGO on CT scans (AIP pattern) and the abnormalities are pathologically cor-
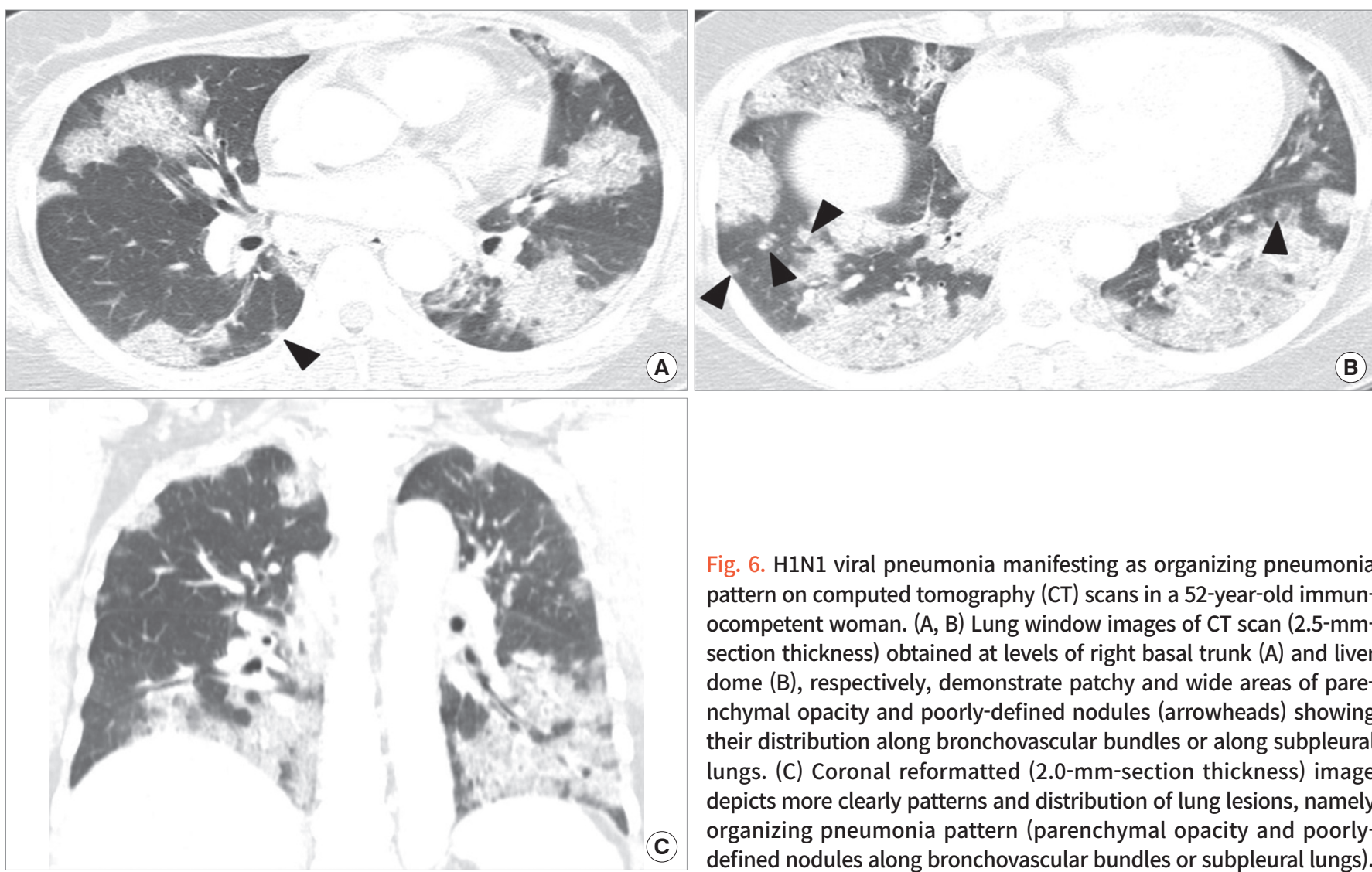

Fig. 6. H1N1 viral pneumonia manifesting as organizing pneumonia pattern on computed tomography (CT) scans in a 52-year-old immunocompetent woman. (A, B) Lung window images of CT scan (2.5- $\mathrm{mm}^{-}$ section thickness) obtained at levels of right basal trunk (A) and liver dome (B), respectively, demonstrate patchy and wide areas of parenchymal opacity and poorly-defined nodules (arrowheads) showing their distribution along bronchovascular bundles or along subpleural lungs. (C) Coronal reformatted (2.0-mm-section thickness) image depicts more clearly patterns and distribution of lung lesions, namely organizing pneumonia pattern (parenchymal opacity and poorlydefined nodules along bronchovascular bundles or subpleural lungs). 
related with diffuse alveolar damage [18]. Therefore, it seems that pattern and extent of lung lesions observed at CT in viral pneumonias may be a determining factor for predicting patient's clinical course and prognosis [8].

The radiologic findings of adult viral pneumonias are variable and overlapping (Table 1). Specific organism diagnosis of a viral pneumonia cannot be made on the basis of imaging features alone. Clinical features such as patient age, immune status, time of year, illness in other family members, community outbreaks, onset, severity, duration of symptoms, and the presence of a rash remain as important aids in diagnosing viral causes of both atypical pneumonia and pneumonia in immunocompromised patients [12].

Table 1. Summary of computed tomography findings in viral pneumonias

\begin{tabular}{|c|c|c|c|c|c|}
\hline & $\begin{array}{l}\text { Centrilobular } \\
\text { nodules }\end{array}$ & $\begin{array}{l}\text { GGO with lobular } \\
\text { distribution }\end{array}$ & $\begin{array}{l}\text { Segmental } \\
\text { consolidation }\end{array}$ & $\begin{array}{c}\text { Thickened } \\
\text { interlobular septae }\end{array}$ & Diffuse GGO \\
\hline Influenza & +++ & +++ & & & + \\
\hline Measles & ++ & + & + & + & + \\
\hline Hantavirus & & & ++ & + & +++ \\
\hline Adenovirus & ++ & + & +++ & & \\
\hline HSV & + & +++ & +++ & & + \\
\hline VZV & +++ & + & & & \\
\hline CMV & ++ & ++ & + & + & ++ \\
\hline EBV & + & + & + & & + \\
\hline
\end{tabular}

Reprinted from Kim et al., with permission from Radiological Society of North America [5].

GGO, ground-glass opacity; HSV, herpes simplex virus; VZV, varicella-zoster virus; CMV, cytomegalovirus; EBV, Epstein-Bar virus.
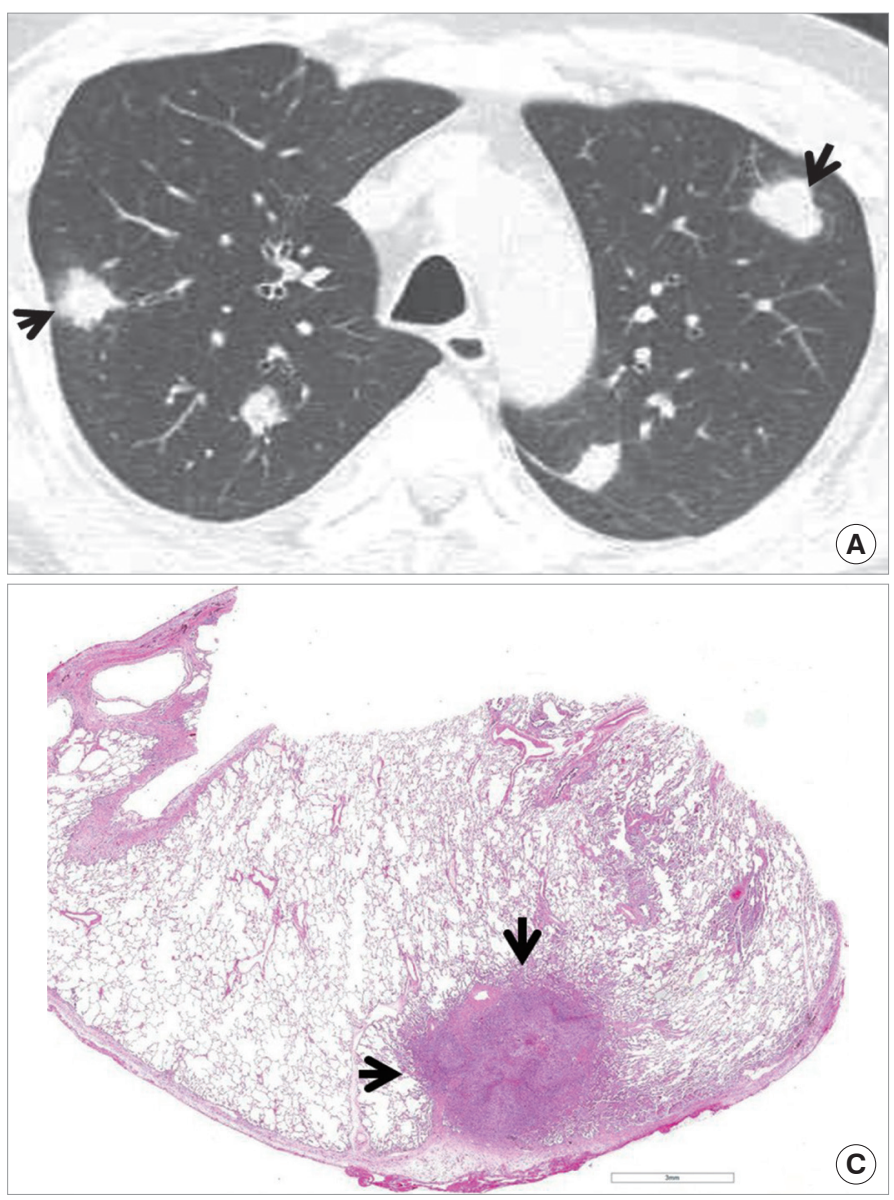

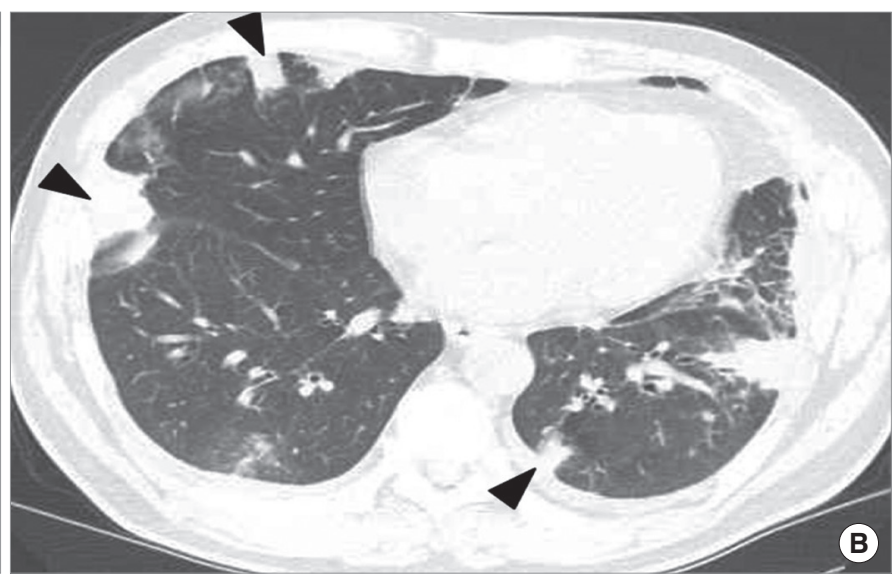

Fig. 7. Septic lung manifesting as multiple peripheral nodules or muItifocal areas of consolidation in both lungs in a 47-year-old man. (A, B) Lung window images of computed tomography scans $(2.5-\mathrm{mm}-$ section thickness) obtained at levels of aortic arch (A) and segmental bronchi in lower lobe (B), respectively, demonstrate multiple variablesized nodules (arrows in A) and areas of variable-sized (acinus or lobule size) consolidation (arrowheads in B) in both lungs. (C) Lowmagnification photomicrograph (H\&E stain) of pathologic specimen obtained from right upper lobe using video-assisted thoracoscopic surgery disclose organizing abscess nodule (arrows). 


\section{MIXED PNEUMONIA}

\section{Septic pneumonia}

The source of infectious emboli includes infected cardiac valves, septic thrombophlebitis, and infected venous catheter or pacemaker. The pneumonia is caused by the obstruction of pulmonary arteries with infected emboli.
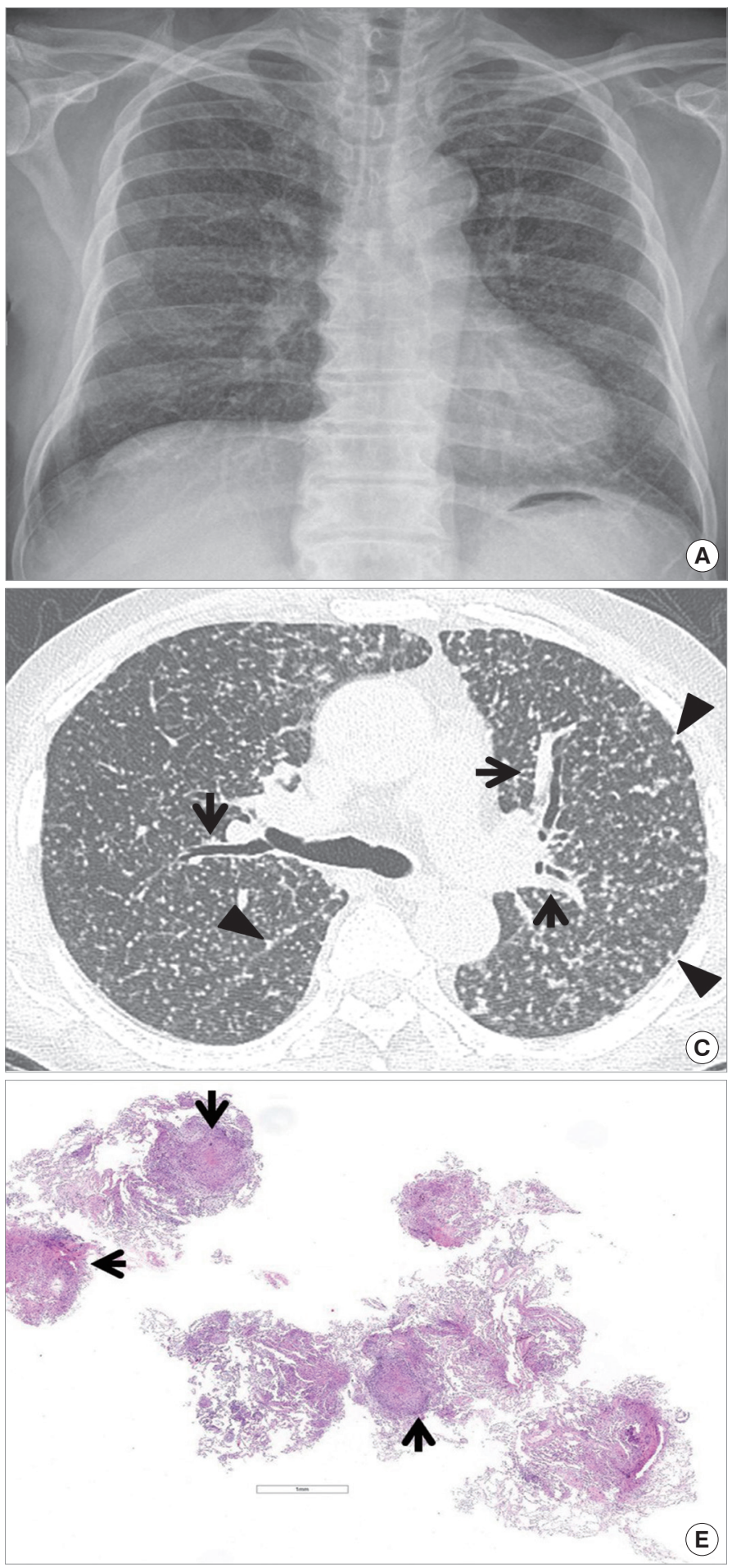

CT findings of septic pneumonia consist of multiple subpleural nodules of 5 to $30 \mathrm{~mm}$ in diameter, feeding vessel sign (trace of vessels to the parenchymal lesions [usually nodules]), cavitation of the nodules, air-bronchogram signs within the nodules, and pleural extension of the lesions with effusion (Fig. 7).

K. pneumoniae, S. aureus, and Streptococcus viridans are
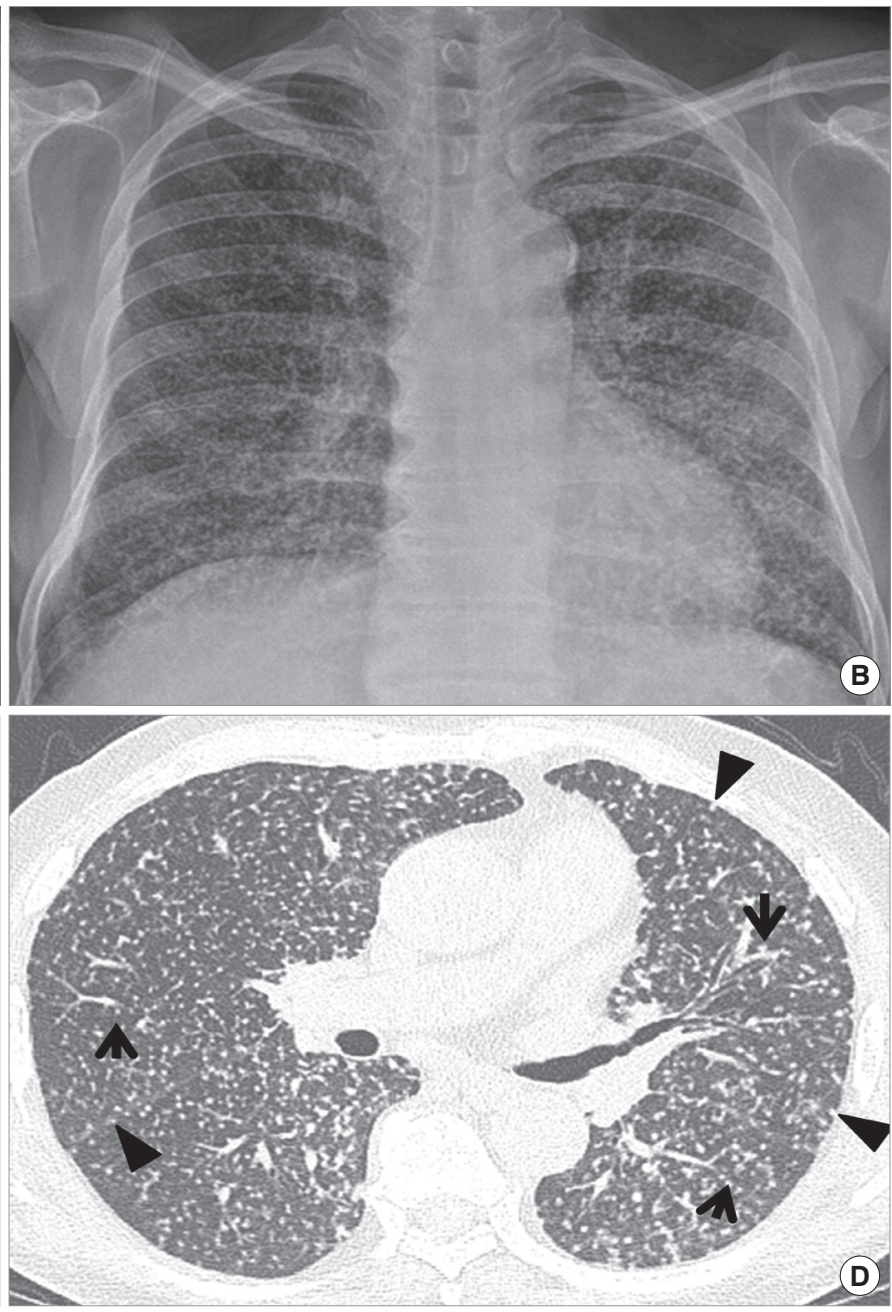

Fig. 8. Miliary tuberculosis in a 71-year-old man. (A) Chest radiograph obtained in April 2016 shows small millet-sized (1 to $3 \mathrm{~mm}$ in diameter) nodules in both lungs. (B) Follow-up radiograph obtained in June 2016 demonstrates that millet-sized nodules have become larger and coarser. (C, D) Lung window images of computed tomography scans (1.5-mm-section thickness) obtained at levels of right upper lobar bronchus (C) and bronchus intermedius (D), respectively, depicts small millet-sized relatively well-defined nodules involving the whole lungs with random distribution, i.e., distributed along the pleurae and fissures (arrowheads), bronchovascular bundles (arrows) and haphazardly in the lung parenchyma. (E) Low-magnification photomicrograph (H\&E stain) of pathologic lung specimen obtained via transbronchial lung biopsy disclose multiple granulomas (arrows) showing internal caseation necrosis. 
common organisms isolated in septic pneumonia. Even though the pathogens of septic pneumonia are diverse depending on the primary foci of infection, early diagnosis and prompt antimicrobial therapy can result in successful treatment outcome [19].

\section{Miliary infection}

Miliary infection of the lung occurs by seeding of microorganisms present in the blood; free microorganisms per se rather than those in fragments of thrombus as in septic pneumonia. Because the microorganisms are minute in size, they lodge within pulmonary capillaries which are present throughout the whole lung. Spread of the microorganisms to the adjacent alveoli and consequent inflammatory reaction tend to demonstrate the distribution of infection randomly in the lung parenchyma (Fig. 8).

The most common organism responsible for a miliary pattern is Mycobacterium tuberculosis. The small granulomas developing in relation to the minute foci of infection tend to be well delineated, resulting in a distinct nodular appearance both pathologically and radiologically (Fig. 8). The characteristic imaging findings consist of nodule, 1 to $3 \mathrm{~mm}$ in diameter, randomly distributed throughout both lungs $[20,21]$.

\section{Lung abscess}

Lung abscess is defined as a liquefactive necrotic lung tissue caused by microbial infection where cavities (usually greater than $20 \mathrm{~mm}$ in diameter) are contained with necrotic debris and fluid (Fig. 9). The sources of microbial infection include the aspiration of oral flora, necrosis within antecedent pneumonia, central malignancy causing obstructive pneumonia, septic emboli, and penetrating trauma. The common locations of lung abscess are the posterior segment of right upper lobe, superior segment of the right lower lobe, superior segment of the left lower lobe and the posterior basal segment of the left lower lobe (Fig. 9).
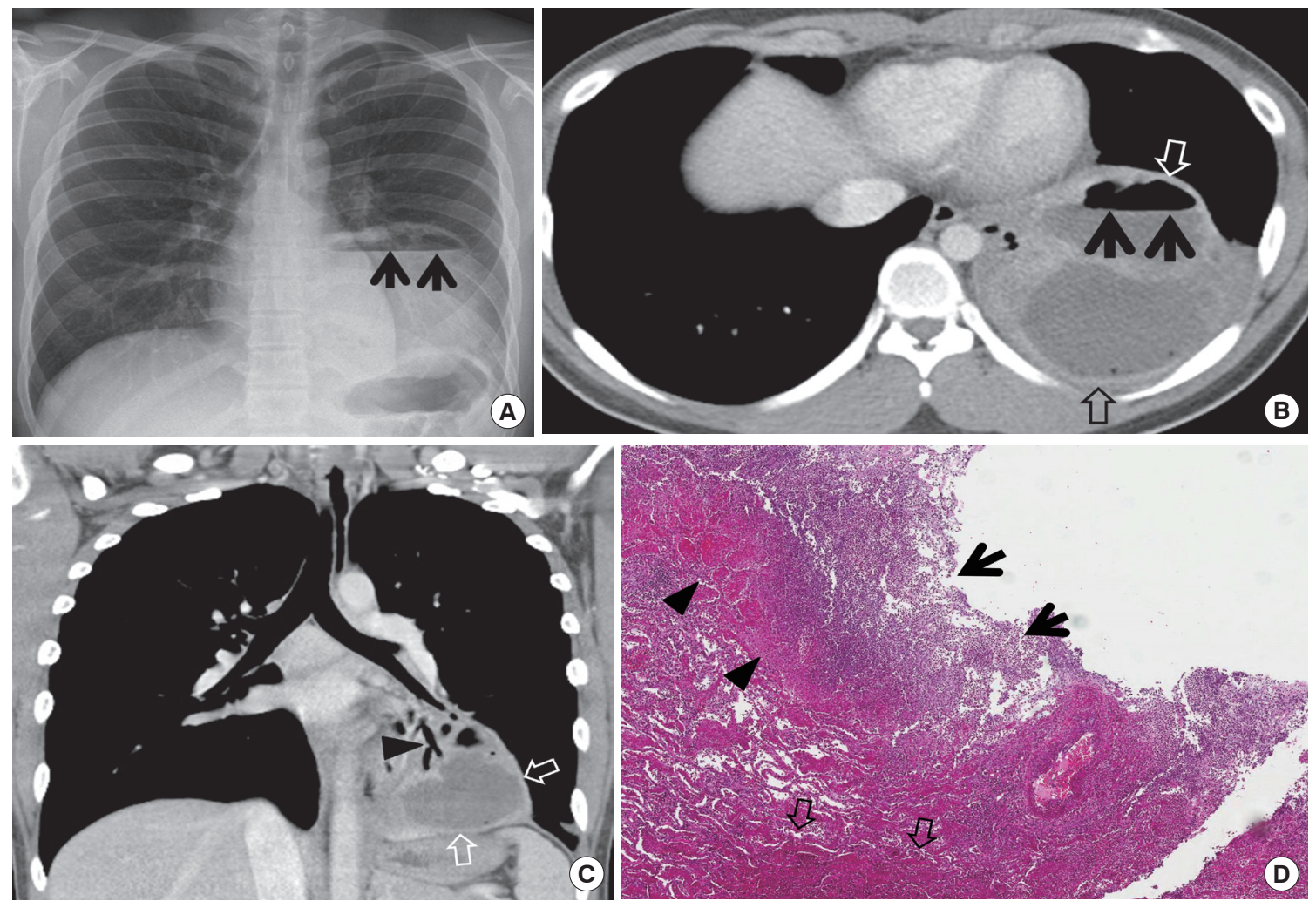

Fig. 9. Lung abscess in a 21-year-old man. (A) Chest radiograph shows a large mass in left lower lung zone containing air-fluid level (arrows) within the lesion. (B) Mediastinal window image of computed tomography (CT) scan (2.5-mm-section thickness) obtained at level of liver dome demonstrates multiloculated cavitary lesion having enhancing wall (open arrows) and internal air-fluid level (arrows) within the lesion. (C) Coronal reformatted (2.0-mm-section thickness) image depicts abscess cavity having enhancing wall (open arrows). Also note surrounding area of consolidation containing CT air-bronchogram sign (arrowhead) within it medial to the cavitary lesion. (D) High-magnification of pathologic specimen (H\&E stain) obtained from necrotizing bronchopneumonia with abscess discloses neutrophilic abscess (arrows), fibrin and necrotic debris (arrowheads) and consolidation in surrounding lung (open arrows) from inner to outer aspect of the cavitary lesion. 
Gross pathology shows central suppuration and surrounding wall of fibrous and granulation tissue. On CT, the abscess presents as a cavity with irregular and thick wall (Fig. 9). The cavity wall shows enhancement supplied by the bronchial artery on enhanced scans. The cavity may rupture into the pleural cavity with resultant pleural effusion (usually multiloculated).

In pre-antibiotic era, anaerobes were found in up to $90 \%$ of cases, but nowadays they are much less frequent. Aerobic bacteria such as Staphylococcus, Klebsiella, and Hemophilus are also frequently involved. Broad-spectrum antibiotics to cover mixed flora is the mainstay of treatment. Most cases respond to antibiotics and prognosis is usually excellent unless there is a debilitating underlying condition. Mortality from lung abscess alone is around $5 \%$ and is improving.

\section{Focal organizing pneumonia}

Focal OP is defined as unresolving pneumonia or pneumonia with delayed resolution. With antimicrobial treatment, pneumonia usually resolves within 4 weeks after its initial manifestation on chest radiograph. However, in about 5\% to $10 \%$ of patients, the pneumonia remains unresolved [22].

Focal OP appears as a nodule or mass, ranging in their diameter from 9 to $66 \mathrm{~mm}$, with various (smooth, lobulated, or lobulated and speculated) contours (Fig. 10). The focal OP may simulate a lung cancer with diverse appearances on CT [23].

\section{Chronic pneumonia}

Chronic pneumonia is an illness that lasts at least 6 weeks and is caused by a microorganism. The pneumonias are usually caused by slow-glowing organisms, such as fungi or mycobacteria. Occasionally, chronic pneumonias cannot be diagnosed, even when lung biopsy specimens ware cultured or studied histopathologically [24].

\section{Cryptococcosis}

Cryptococcus neoformans is a ubiquitous encapsulated yeastlike fungus that is found worldwide; particularly in soils that are contaminated with pigeon excreta and decayed wood [25]. Inhalation of cryptococcal particles into the lungs is a route of pulmonary infection, and subsequent hematoge-
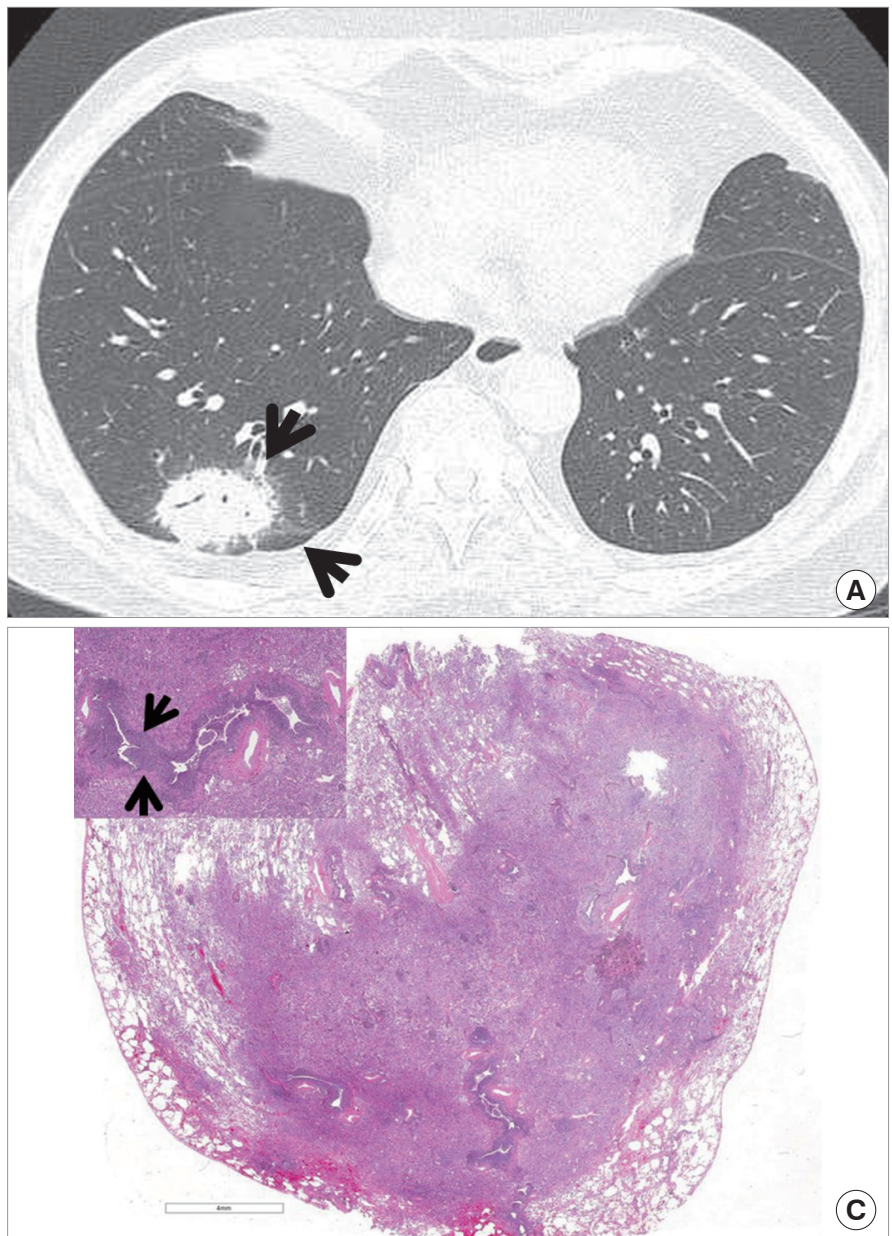

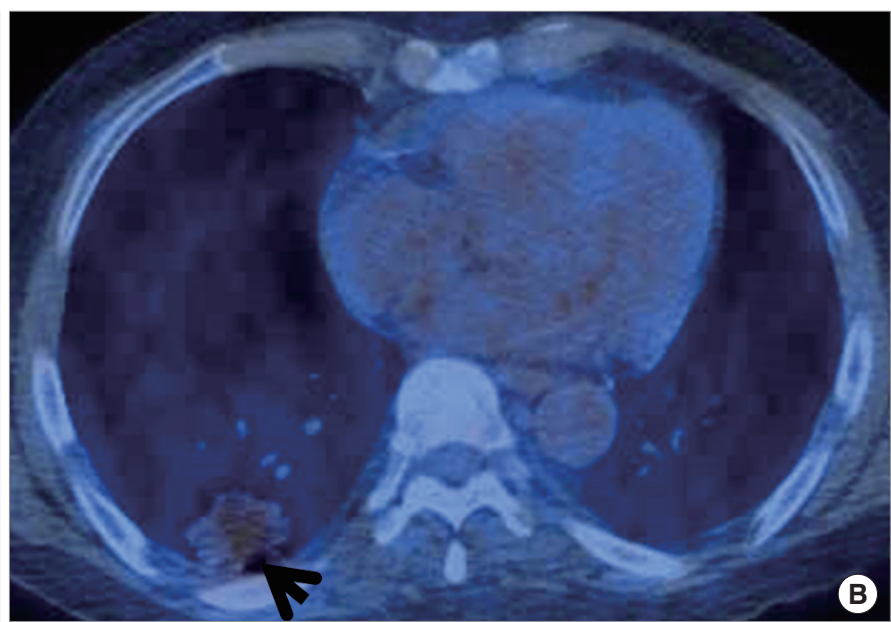

Fig. 10. Organizing pneumonia in a 69-year-old man. (A) Lung window image of computed tomography (CT) scan (2.5-mm-section thickness) obtained at level of supra-hepatic inferior vena cava sows an oval soft tissue mass surrounded by ground-glass opacity (arrows) in right lower lobe. (B) Fludeoxyglucose (FDG) positron emission tomography /CT obtained at similar level to (A) demonstrates similar FDG uptake (arrow) to mediastinal blood pool uptake, suggesting rather benign nature of the lesion. (C) Low-magnification of photomicrograph (H\&E stain) of pathologic specimen obtained with video-assisted thoracoscopic surgery discloses consolidative lung lesion comprised of varying extent of organizing fibrosis and chronic inflammation (inset: airway walls showing peribronchiolar lymphocyte infiltration [arrows in inset] without cellular uniformity). 
nous dissemination may cause central nervous system infection $[25,26]$.

Pulmonary cryptococcosis is an indolent lung disease in non-AIDS patients that is slowly progressive in nature, even without adequate treatment. Moreover, it does not show a rapid resolution of the lung abnormalities even with antifungal therapy.

The disease may appear with various patterns of lung lesions; however, a multiple clustered-nodular pattern, localized to a lung lobe, is the most common abnormality (Fig. 11). In half of the cases, the disease appeared in patients with underlying disease, in which most of the patients have an extra-thoracic malignant condition. Therefore, when lung le- sions show clustered or scattered nodules of an indolent nature, pulmonary cryptococcosis should be considered in the differential diagnosis, even for a patient with an extrathoracic malignancy. The lesions may show high ${ }^{18} \mathrm{~F}$-fludeoxyglucose $\left({ }^{18} \mathrm{~F}-\mathrm{FDG}\right)$ uptake and may thus simulate a malignant lung condition at positron emission tomography (PET). Although clinical improvement can be achieved in all patients, radiological improvement is slow, even after antifungal therapy. Moreover, lung lesions may persist for a long time, showing a divergence from the clinical response [25].

\section{Actinomycosis}

Thoracic actinomycosis is a chronic suppurative pulmonary
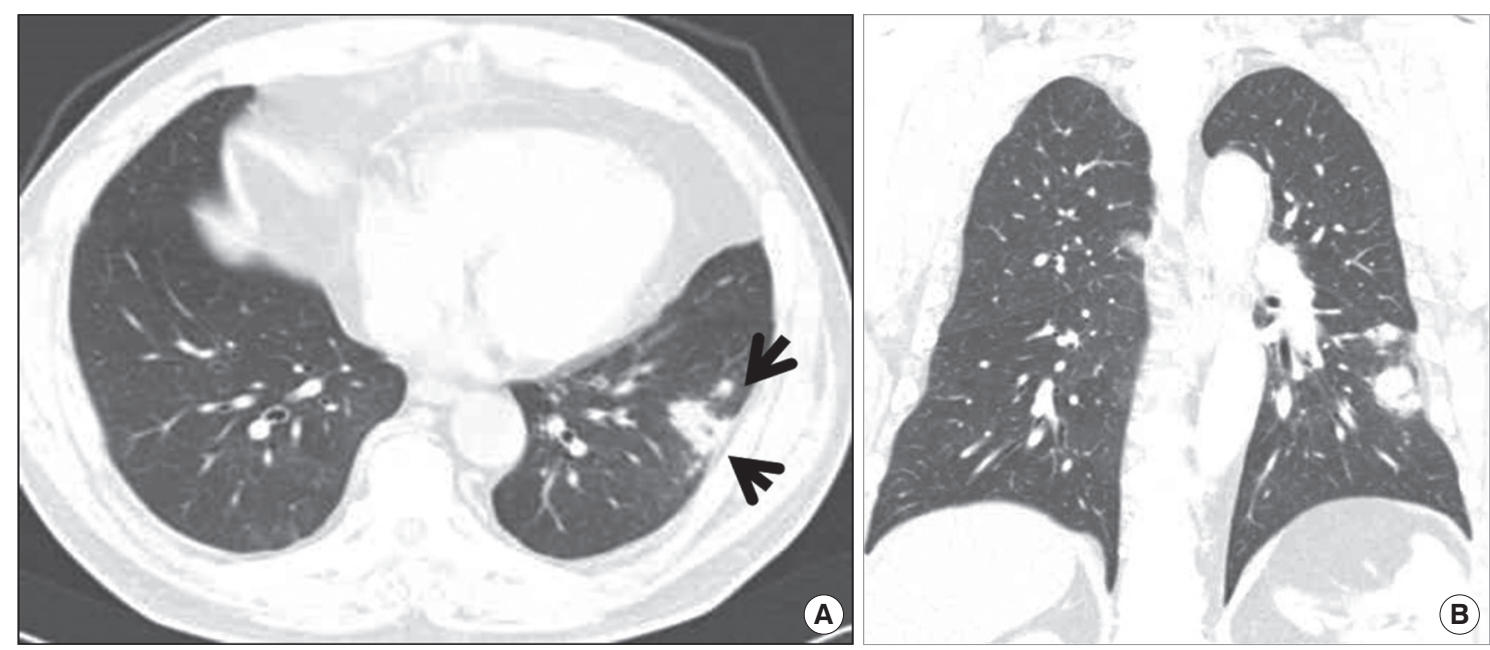

Fig. 11. Cryptococcosis in a 60-year-old immunocompetent man. (A) Lung window image of computed tomography scans (2.5-mm-section thickness) obtained at level of segmental bronchi shows poorly-defined nodules (arrows) in left lower lobe. (B) Coronal-reformatted (2.0-mm-section thickness) image demonstrates nodular clustering in superior segment of left lower lobe.
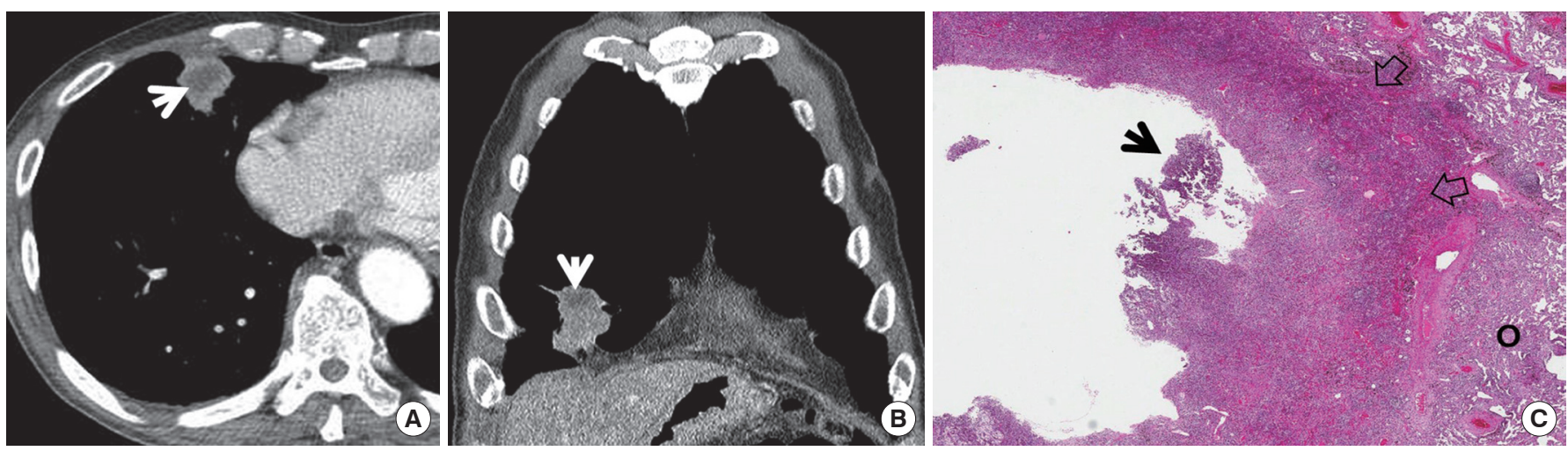

Fig. 12. Actinomycosis in an 80-year-old man. (A) Mediastinal window image of computed tomography (CT) (2.5-mm-section thickness) scan obtained at levels of cardiac ventricle shows a nodule harboring central geographic necrosis (arrow) in right middle lobe. (B) Coronalreformatted (2.0-mm-section thickness) CT image demonstrates same lesion containing necrotic area (arrow). (C) High-magnification photomicrograph (H\&E stain) of surgical specimen obtained with video-assisted thoracoscopic surgery discloses abscess having basophilic material within cavity (proved to be sulfur granule with special staining, arrow). Also note cavity wall (open arrows) and surrounding organizing pneumonia (circle). 

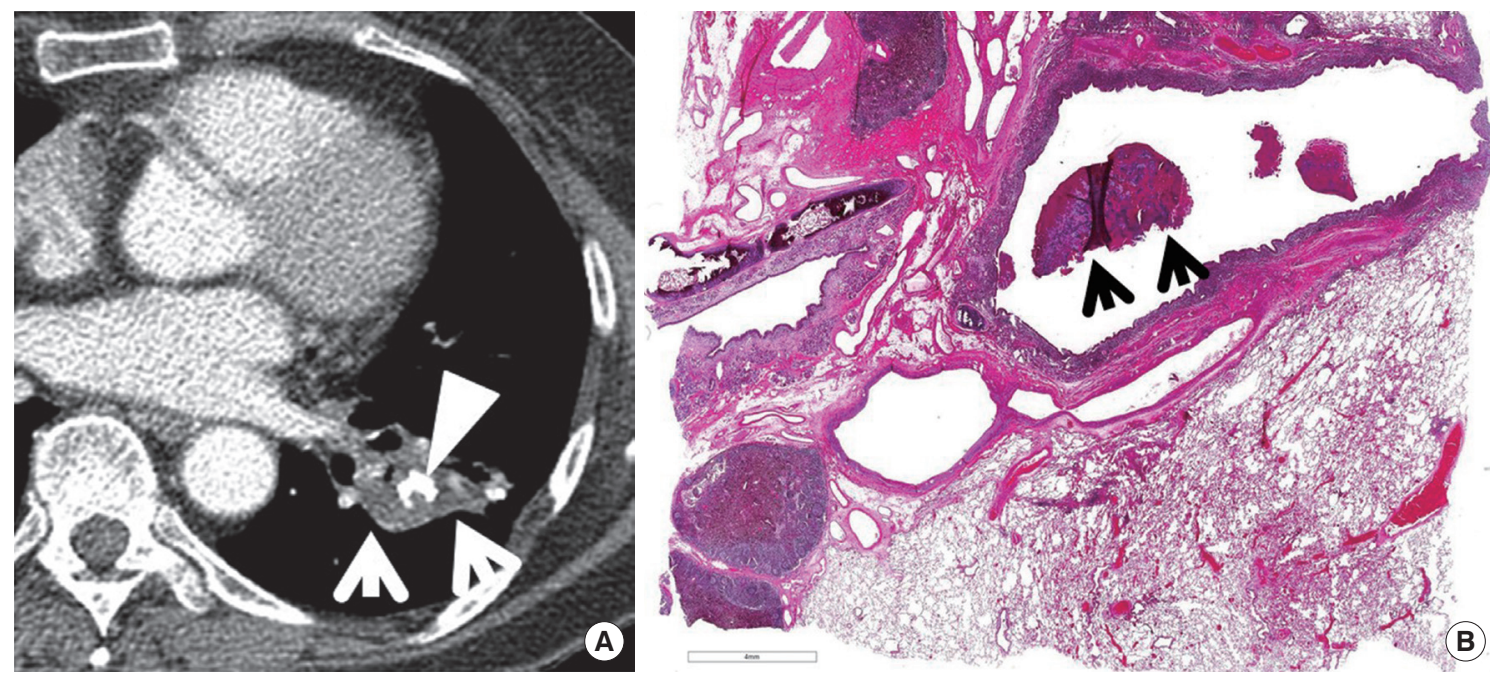

Fig. 13. Bronchial actinomycosis associated with broncholithiasis in a 70-year-old woman. (A) Mediastinal window image of computed tomography scan (2.5-mm-section thickness) obtained at level of left atrium shows low-attenuation soft-tissue lesion (arrows) containing calcific attenuation (arrowhead) within it in left lower lobe. (B) Low-magnification photomicrograph (H\&E stain) of surgical specimen obtained with video-assisted thoracoscopic surgery discloses dilated bronchus harboring a broncholith covered with inflammatory cell exudate and bacterial colonies with sulfur granule (arrows).

Table 2. Diagnostic criteria of CNPA and CCPA

\begin{tabular}{lll}
\hline \multicolumn{1}{c}{ CNPA } & CCPA \\
\hline Clinical & $\begin{array}{c}\text { 1-3-month history of chronic pulmonary symptoms } \\
\text { or chronic illness }\end{array}$ & $\begin{array}{c}\text { Three month history of chronic pulmonary symptoms } \\
\text { or chronic illness }\end{array}$ \\
Radiological & $\begin{array}{c}\text { Chronic parenchymal opacity, subsequent progressive } \\
\text { cavitation, and consequent aspergilloma within cavity }\end{array}$ & $\begin{array}{c}\text { Progressive radiological abnormalities, with cavity, pleural thickening, } \\
\text { pericavitary infiltrates, and sometimes a fungus ball }\end{array}$ \\
Laboratory & $\begin{array}{c}\text { Positive Aspergillus galactomannan antigen in blood } \\
\text { or respiratory fluid }\end{array}$ & Aspergillus lgG antibody elevated, or other microbiological data \\
& and
\end{tabular}

Based on Infectious Diseases Society of America (IDSA) criteria.

CNPA, chronic necrotizing pulmonary aspergillosis; CCPA, chronic cavitary pulmonary aspergillosis; IgG, immunoglobulin G.

or endobronchial infection caused by Actinomyces israelii, a gram-positive anaerobic organism. Actinomyces infection typically follows aspiratin of endogenous organisms of the oropharynx into the lungs in persons with poor oral hygiene or from extension of cervicofacial infection. The clinical manifestations of pulmonary actinomycosis are productive cough, low-grade fever, and blood tinged sputum. The prognosis is generally good, provided that the infection is recognized and appropriate antibiotic therapy is instituted [27].

The typical CT feature of parenchymal actinomycosis is a chronic segmental air-space consolidation containing necrotic low-attenuation areas with frequent cavity formation (Fig. 12) [27]. A broncholith can be secondarily infected with Actinomyces organisms, resulting in endobronchial actinomycosis. It usually manifests as a proximal endobronchial calcification associated with distal obstructive pneumonia (Fig. 13) [28].

\section{Chronic pulmonary aspergillosis}

The Infectious Diseases Society of America (IDSA) have proposed three major subtypes of chronic forms of pulmonary aspergillosis, namely chronic necrotizing pulmonary aspergillosis (CNPA; categorized in subacute invasive form of aspergillosis or subacute invasive pulmonary aspergillosis), chronic cavitary pulmonary aspergillosis (CCPA), and aspergilloma [29]. Updated IDSA guidelines have indicated that the differences between CNPA and CCPA include prolonged time frame (CNPA, 1 to 3 months vs. CCPA, > 3 months) (Table 2).

When chronic parenchymal opacity, subsequent progressive cavitation, and consequent aspergilloma formation are serially seen, the diagnosis of CNPA may be made (Fig. 14). Conversely, when pre-existing cavities are subsequently being surrounded by peri-cavitary opacity with or without aspergilloma, the diagnosis of CCPA could be made [30]. 

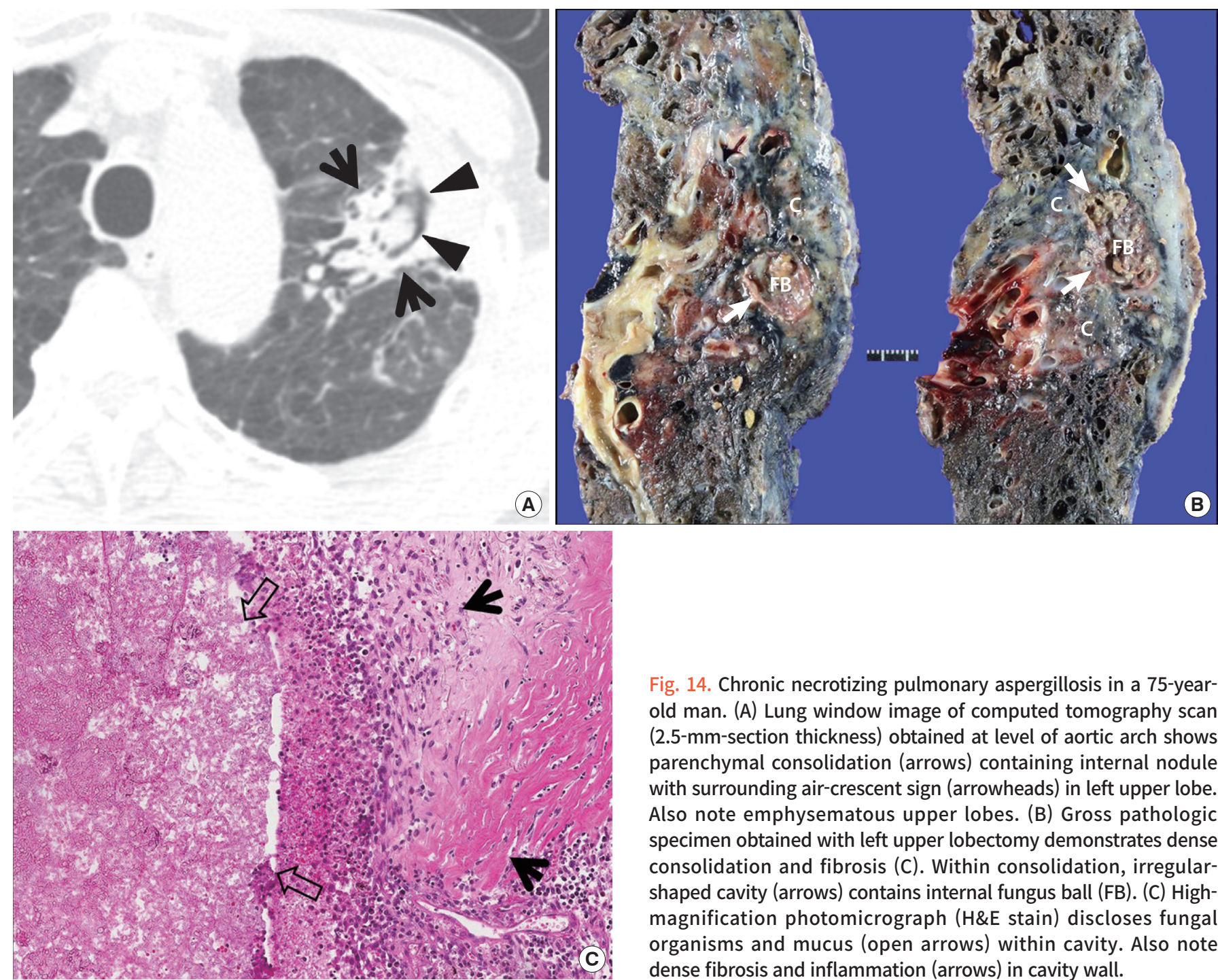

Fig. 14. Chronic necrotizing pulmonary aspergillosis in a 75-yearold man. (A) Lung window image of computed tomography scan (2.5-mm-section thickness) obtained at level of aortic arch shows parenchymal consolidation (arrows) containing internal nodule with surrounding air-crescent sign (arrowheads) in left upper lobe. Also note emphysematous upper lobes. (B) Gross pathologic specimen obtained with left upper lobectomy demonstrates dense consolidation and fibrosis (C). Within consolidation, irregularshaped cavity (arrows) contains internal fungus ball (FB). (C) Highmagnification photomicrograph (H\&E stain) discloses fungal organisms and mucus (open arrows) within cavity. Also note dense fibrosis and inflammation (arrows) in cavity wall.

Patients with chronic pulmonary aspergillosis are 60 years in their mean age (interquartile range, 45 to 65 years), and show male predominance (approximately $80 \%$ ). The most common underlying lung disease is pulmonary TB that is seen in more than $90 \%$ of the patients [31].

\section{THE ROLE OF IMAGING IN PNEUMONIA}

The main role of imaging lies in the detection or exclusion of the presence of pneumonia. Other roles include narrowing down of differential diagnoses of pneumonia regarding causative organisms involved. Further diagnostic approach may be enlightened by observing initial imaging findings. The response to antibiotic therapy may be evaluated by observing follow-up imaging studies.

\section{ANTIBIOTIC THERAPY}

Etiologic diagnosis is important for choosing appropriate antibiotics to treat pneumonia. Etiologic diagnosis is often difficult even though the development of imaging and microbiologic diagnostic techniques. Antibiotic treatment should be empirically started against pathogens presumed based on clinical manifestations and imaging patterns before microbiologic results are available. The current paradigm for management of serious hospital-associated infections is to initiate empiric therapy with a broad-spectrum regimen covering likely pathogens, based on local surveillance and susceptibility data, and presence of risk factors for involvement of a resistant microorganism. Subsequent modification (de-escalation) of the initial regimen become possible later, when culture results are available and clinical status can be better as- 
sessed, 2 to 4 days after initiation of empiric therapy [32].

Ampicillin, amoxicillin, ceftriaxone, cefotaxime, and respiratory fluoroquinolones (levofloxacin, moxifloxacin) are recommended treatment agents for S. pneumoniae, the most common pathogen causing CAP. Macrolides (azithromycin, clarithromycin) and respiratory fluoroquinolones are treatment options for atypical pneumonia [33]. When fluoroquinolone (a critical component of antituberculous drug regimens for multidrug-resistant TB) is selected, the possibility of TB should be excluded, because exposure to fluoroquinolone prior to the diagnosis of TB has been associated with fluoroquinolone resistance, particularly when the fluoroquinolone exposure occurs $>60$ days before TB diagnosis and for longer than 10 days [34].

New antibiotics having activity against pathogens resistant to conventional antibiotics have been developed: anti-MRSA (methicillin-resistant S. aureus) cephalosporins (ceftaroline, ceftobiprole), $\beta$-lactams active against resistant Pseudomonas (ceftolozane/tazobactam, ceftazidime/avibactam), new carbapenems (tomopenem, razupenem, imipenem/relebactam), a novel aminomethylcycline agent (omadacycline), etc. Intrapulmonary penetration of antibiotics should be considered in the management of pneumonia. Daptomycin is well known not to be used to treat pneumonia owing to the difficulty of lung penetration of the antibiotics [35]. Regardless of initial antibiotics, coverage should be narrowed down on the basis of susceptibility test results of isolated pathogens.

The conventional 10- to 14-day treatment duration is anecdotal patterns of behavior, not supported by evidence. Recent guidelines recommend 5- to 7-day treatment based on studies showing that a period shorter than 7 days was as effective as longer duration of therapy for mild to moderate CAP [33]. Many adjunctive therapeutic agents including corticosteroids, statins, immunoglobulins, granulocyte colony-stimulating factors, probiotics, and anticoagulants did not show a significant role in pneumonia therapy.

For the viral pneumonia, supportive management is the main therapy. Since several specific antiviral agents are available (oseltamivir or peramivir for influenza, ribavirin for respiratory syncytial virus [RSV], and cidofovir for adenovirus), detection of these viruses is important [36]. Studies on the efficacy of novel antiviral drugs are ongoing.

\section{CONCLUSION}

The imaging features of pneumonias caused by various organisms are overlapping. Morphologic classification of pneu- monia may help differentiate pneumonias induced by diverse organisms. CT clearly demonstrates the presence of pneumonia and help morphologic classification of the pneumonia. HAP present with complicated imaging findings and should be differentiated from various lung conditions. Chronic indolent nature both in evolution and improvement may suggest the presence of chronic pneumonia such as cryptococcosis, actinomycosis, or CNPA or CCPA. Invasive diagnostic procedure may be needed to make a definite diagnosis of pneumonia and its causative organisms.

New antibiotics having activity against pathogens resistant to conventional antibiotics have been developed. The conventional 10- to 14-day treatment duration is anecdotal patterns of behavior, and recent guidelines recommend 5- to 7-day treatment for mild to moderate CAP with evidencebased studies. Since several specific antiviral agents are available (oseltamivir or peramivir for influenza, ribavirin for RSV, and cidofovir for adenovirus), detection of these viruses is important.

\section{CONFLICTS OF INTEREST}

No potential conflict of interest relevant to this article was reported.

\section{REFERENCES}

1. Ruuskanen O, Lahti E, Jennings LC, Murdoch DR. Viral pneumonia. Lancet 2011;377:1264-75.

2. Nair GB, Niederman MS. Community-acquired pneumonia: an unfinished battle. Med Clin North Am 2011;95:1143-61.

3. Anand N, Kollef MH. The alphabet soup of pneumonia: CAP, HAP, HCAP, NHAP, and VAP. Semin Respir Crit Care Med 2009;30:3-9.

4. American Thoracic Society; Infectious Diseases Society of America. Guidelines for the management of adults with hospital-acquired, ventilator-associated, and healthcareassociated pneumonia. Am J Respir Crit Care Med 2005; 171:388-416

5. Craven DE. What is healthcare-associated pneumonia, and how should it be treated? Curr Opin Infect Dis 2006; 19:153-60

6. Brown PD, Lerner SA. Community-acquired pneumonia. Lancet 1998;352:1295-302.

7. Logroscino CD, Penza O, Locicero S, Losito G, Nardini S, Bertoli L, et al. Community-acquired pneumonia in adults: a multicentric observational AIPO study. Monaldi 
Arch Chest Dis 1999;54:11-7.

8. Tanaka N, Matsumoto T, Kuramitsu T, Nakaki H, Ito K, Uchisako $\mathrm{H}$, et al. High resolution CT findings in community-acquired pneumonia. J Comput Assist Tomogr 1996; 20:600-8.

9. Lee JY, Lee KS, Jung KJ, Han J, Kwon OJ, Kim J, et al. Pulmonary tuberculosis: CT and pathologic correlation. J Comput Assist Tomogr 2000;24:691-8.

10. Ikezoe J, Takeuchi N, Johkoh T, Kohno N, Tomiyama N, Kozuka T, et al. CT appearance of pulmonary tuberculosis in diabetic and immunocompromised patients: comparison with patients who had no underlying disease. AJR Am J Roentgenol 1992;159:1175-9.

11. Reittner P, Muller NL, Heyneman L, Johkoh T, Park JS, Lee $\mathrm{KS}$, et al. Mycoplasma pneumoniae pneumonia: radiographic and high-resolution CT features in 28 patients. AJR Am J Roentgenol 2000;174:37-41.

12. Kim EA, Lee KS, Primack SL, Yoon HK, Byun HS, Kim TS, et al. Viral pneumonias in adults: radiologic and pathologic findings. Radiographics 2002;22 Spec No:S137-49.

13. Han BK, Son JA, Yoon HK, Lee SI. Epidemic adenoviral lower respiratory tract infection in pediatric patients: radiographic and clinical characteristics. AJR Am J Roentgenol 1998;170:1077-80.

14. Moon JH, Kim EA, Lee KS, Kim TS, Jung KJ, Song JH. Cytomegalovirus pneumonia: high-resolution CT findings in ten non-AIDS immunocompromised patients. Korean J Radiol 2000;1:73-8.

15. Miller WT Jr, Mickus TJ, Barbosa E Jr, Mullin C, Van Deerlin VM, Shiley KT. CT of viral lower respiratory tract infections in adults: comparison among viral organisms and between viral and bacterial infections. AJR Am J Roentgenol 2011;197:1088-95.

16. Shiley KT, Van Deerlin VM, Miller WT Jr. Chest CT features of community-acquired respiratory viral infections in adult inpatients with lower respiratory tract infections. J Thorac Imaging 2010;25:68-75.

17. Kim SY, Kim JS, Park CS. Various computed tomography findings of $2009 \mathrm{H} 1 \mathrm{~N} 1$ influenza in 17 patients with relatively mild illness. Jpn J Radiol 2011;29:301-6.

18. Marchiori E, Zanetti G, Fontes CA, Santos ML, Valiante PM, Mano CM, et al. Influenza A (H1N1) virus-associated pneumonia: high-resolution computed tomographypathologic correlation. Eur J Radiol 2011;80:e500-4.

19. Lee SJ, Cha SI, Kim CH, Park JY, Jung TH, Jeon KN, et al. Septic pulmonary embolism in Korea: microbiology, clinicoradiologic features, and treatment outcome. J In- fect 2007;54:230-4.

20. Jeong YJ, Lee KS. Pulmonary tuberculosis: up-to-date imaging and management. AJR Am J Roentgenol 2008; 191:834-44.

21. McGuinness G, Naidich DP, Jagirdar J, Leitman B, McCauley DI. High resolution CT findings in miliary lung disease. J Comput Assist Tomogr 1992;16:384-90.

22. Huo Z, Feng R, Tian X, Zhang H, Huo L, Liu H. Clinicopathological findings of focal organizing pneumonia: a retrospective study of 37 cases. Int J Clin Exp Pathol 2015;8:511-6.

23. Yang PS, Lee KS, Han J, Kim EA, Kim TS, Choo IW. Focal organizing pneumonia: CT and pathologic findings. J Korean Med Sci 2001;16:573-8.

24. Geppert EF. Chronic and recurrent pneumonia. Semin Respir Infect 1992;7:282-8.

25. Song KD, Lee KS, Chung MP, Kwon OJ, Kim TS, Yi CA, et al. Pulmonary cryptococcosis: imaging findings in 23 nonAIDS patients. Korean J Radiol 2010;11:407-16.

26. Sarosi GA. Cryptococcal pneumonia. Semin Respir Infect 1997;12:50-3.

27. Kim TS, Han J, Koh WJ, Choi JC, Chung MJ, Lee JH, et al. Thoracic actinomycosis: CT features with histopathologic correlation. AJR Am J Roentgenol 2006;186:225-31.

28. Kim TS, Han J, Koh WJ, Choi JC, Chung MJ, Lee KS, et al. Endobronchial actinomycosis associated with broncholithiasis: CT findings for nine patients. AJR Am J Roentgenol 2005;185:347-53.

29. Walsh TJ, Anaissie EJ, Denning DW, Herbrecht R, Kontoyiannis DP, Marr KA, et al. Treatment of aspergillosis: clinical practice guidelines of the Infectious Diseases Society of America. Clin Infect Dis 2008;46:327-60.

30. Izumikawa K, Tashiro T, Tashiro M, Takazono T, Kosai K, Morinaga $Y$, et al. Pathogenesis and clinical features of chronic pulmonary aspergillosis: is it possible to distinguish CNPA and CCPA clinically? J Infect Chemother 2014;20:208-12.

31. Nam HS, Jeon K, Um SW, Suh GY, Chung MP, Kim H, et al. Clinical characteristics and treatment outcomes of chronic necrotizing pulmonary aspergillosis: a review of 43 cases. Int J Infect Dis 2010;14:e479-82.

32. Snydman DR. Empiric antibiotic selection strategies for healthcare-associated pneumonia, intra-abdominal infections, and catheter-associated bacteremia. J Hosp Med 2012;7 Suppl 1:S2-12.

33. Mandell LA, Wunderink RG, Anzueto A, Bartlett JG, Campbell GD, Dean NC, et al. Infectious Diseases Society of America/American Thoracic Society consensus guide- 
lines on the management of community-acquired pneumonia in adults. Clin Infect Dis 2007;44 Suppl 2:S27-72.

34. Devasia RA, Blackman A, Gebretsadik T, Griffin M, Shintani A, May $\mathrm{C}$, et al. Fluoroquinolone resistance in Mycobacterium tuberculosis: the effect of duration and timing of fluoroquinolone exposure. Am J Respir Crit Care Med 2009;180:365-70.

35. Purrello SM, Garau J, Giamarellos E, Mazzei T, Pea F, Sori- ano A, et al. Methicillin-resistant Staphylococcus aureus infections: a review of the currently available treatment options. J Glob Antimicrob Resist 2016:7:178-86.

36. Pavia AT. What is the role of respiratory viruses in community-acquired pneumonia? What is the best therapy for influenza and other viral causes of community-acquired pneumonia? Infect Dis Clin North Am 2013;27: $157-75$. 